\title{
Guest editorial: Networked television
}

\author{
Cristian Hesselman · Pablo Cesar · David Geerts
}

Published online: 11 August 2010

(C) Springer-Verlag 2010

This special issue on networked television consists of a selection of the best papers of the 7th European Interactive TV Conference (EuroITV 2009), which was held in Leuven, Belgium, in June of 2009. Networked television is a new concept, which is about TV sets becoming yet another node in many IP-based networks, such as managed IPTV networks and in-home networks. This increased level of connectivity allows for new types of services and will eventually result in the seamless integration of interactive TV into the wide range of other services that people have at their disposal. The papers in this special issue consider various challenges to make this vision a reality, such as scalability of catch-up TV services and the integration of interactive TV services and home control automation.

The goal of EuroITV 2009 was to provide a discussion and dissemination platform for topics like these, both for industry and academia, so as to fuel new business and worldclass research in networked television. The conference program consisted of a technical track, a business track, and a track on user studies, embodying EuroITV's unique multidisciplinary approach. The conference acceptance rate was $41 \%$ out of 88 submissions, which illustrates that it is a

C. Hesselman $(\square)$

Novay, P.O. Box 589, 7500AN Enschede, The Netherlands

e-mail: cristian.hesselman@novay.nl

P. Cesar

CWI: Centrum Wiskunde \& Informatica,

Science Park 123, 1098XG Amsterdam,

The Netherlands

e-mail: P.S.Cesar@cwi.nl

D. Geerts

IBBT and K.U. Leuven, Parkstraat 45,

P.O. Box 3605, 3000 Leuven, Belgium

e-mail: david.geerts@soc.kuleuven.be selective and popular event. The presented papers were from researchers and practitioners from Europe, Asia, and the Americas. EuroITV 2009 was attended by a total of 197 people, many of whom also contributed to the pre-conference workshops, the industry track, the tutorials, and the doctoral consortium.

From the papers presented in Leuven, we invited the best six to submit an extended version of their work for this special issue of Multimedia System Journal, with the requirement that they should top up their EuroITV paper with at least $30 \%$ of new results. After a multi-round reviewing process with three or four experts reviewing each paper, we were able to retain three papers, which are the ones that are now part of this special issue.

In the first paper, Avramova, De Vleeschauwer, Wittevrongel, and Bruneel present a rigorous performance analysis of a distributed caching algorithm for catch-up television. The authors developed the algorithm to avoid overloads in managed IPTV networks and evaluated it through extensive simulations. They conclude that their new algorithm clearly outperforms existing caching algorithms and also investigate how to configure the algorithm's parameters for optimal performance.

The second paper, by Viana and de Lucena, is about a software platform that seamlessly integrates interactive TV and home control services. The main advantage of their work is that the platform allows developers to easily write mixed interactive TV-home control applications. Such applications also facilitate new user experiences, such as synchronizing home appliances with the audio/video streams of a movie. The novelty of their work is that the platform transparently combines Ginga middleware technology (for interactive TV) with OSGi (for home control), while at the same time exploiting the declarative part of the Ginga middleware. 
The third paper is by Manzato, Coimbra, and Goularte and discusses a system that helps TV viewers annotate news programs. The system accomplishes this through a novel search and selection facility that dynamically combines different search mechanisms (face recognition, keywords, and visual characteristics of scenes) to find videos that are semantically related to the news program being watched. The authors evaluate the precision of their search and selection algorithm and conclude that it performs better than the individual search mechanisms.

We believe the above three papers cover major challenges in networked television and provide an excellent read for anyone interested in the scientific state of affairs in this field. We hope you will enjoy reading them as much as we did.

\section{Reviewers}

Daniele Abbadessa (NEC, Germany)

Jorge Abreu (University of Aveiro, Portugal)

Shelley Buchinger (University of Vienna, Austria)

Tobias Bürger (Universität Innsbruck, Austria)

Konstantinos Chorianopoulos (Ionian University, Greece)

Oskar van Deventer (TNO, Netherlands)

Oliver Friedrich (Fraunhofer Fokus, Germany)

Alberto Gil (University of Vigo, Spain)

Luiz Fernando Gomes Soares (Pontifícia Universidade

Católica do Rio de Janeiro, Brazil)

Gunnar Harboe (Motorola, USA)

Ian Kegel (British Telecom, UK)

Ralf Kernchen (University of Surrey, UK)

Rodrigo Laiola Guimaraes (CWI: Centrum Wiskunde \& Informatica, Netherlands)

Marianna Obrist (University of Salzburg, Austria)

Andrew Tokmakoff (Philips Research, Netherlands)

Marian Ursu (University of London, UK)

Harry van Vliet (Utrecht University of Applied Sciences, Netherlands)

Christian Wartena (Novay, Netherlands)
Author Biographies

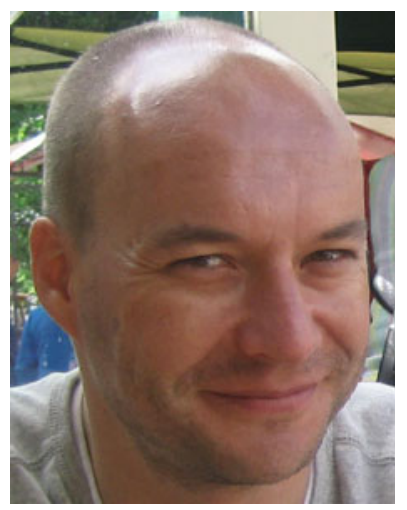

Cristian Hesselman (cristian. hesselman@novay.nl) is a senior researcher at Novay, the Dutch national research centre for applied research on information and communication technologies. His main research interests are converged multimedia systems, adaptive multimodal user interfaces and their integration with the Internet of Things. Cristian also leads research projects in this field, such as Interactive Networked Experiences in Multimedia for You (http://www.inem4u.eu). He has a PhD in computer science from the University of Twente, the Netherlands. Webpage: http://www. lab.telin.nl/ hesselma/. Blog: http://blog.pervasivemedia.nl/.

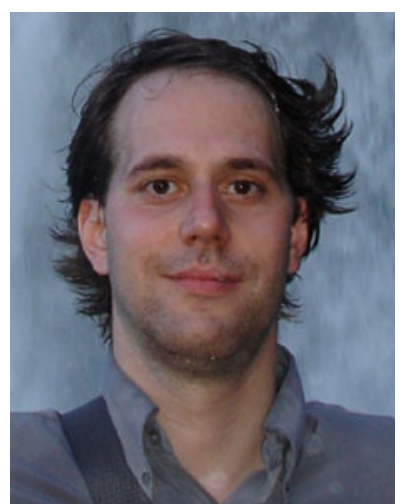

Pablo Cesar (P.S.Cesar@ cwi.nl) is a tenure track researcher in the Distributed Multimedia Languages and Infrastructures research group at CWI (The National Research Institute for Mathematics and Computer Science in the Netherlands). He received his $\mathrm{PhD}$ from the Helsinki University of Technology in 2006. He has (co)authored over 40 articles (conference papers and journal articles) about multimedia systems and infrastructures, social media sharing, interactive media, multimedia content modeling, and user interaction. He is involved in standardization activities (e.g., SMIL from W3C) and has been active in a number of European projects such as Passepartout, SPICE, iNEM4U, and Ta2. He is coeditor of the book "Social Interactive Television: Immersive Shared Experiences and Perspectives" and has given tutorials about multimedia systems in prestigious conferences such as ACM Multimedia and the WWW conference. Webpage: http://homepages.cwi.nl/ $\sim$ garcia/. 


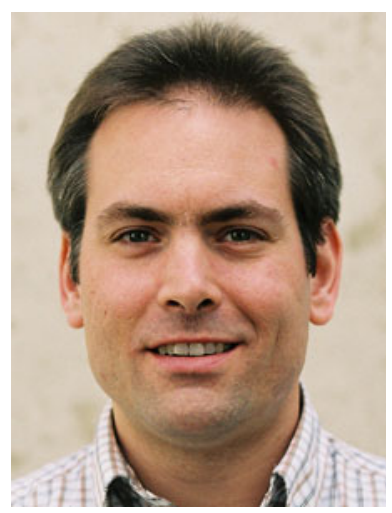

David Geerts (David.Geerts@ soc.kuleuven.be) has a master in Communication Science at the K.U.Leuven, a master in Culture and Communication at the K.U.Brussel and a PhD in Social Sciences at the K.U.Leuven, where he now leads the Centre for User Experience Research (CUO) at the faculty of Social Sciences. David is specialized in user-centered design and evaluation of future ICT applications, such as multi-touch surfaces, social interactive television, serious games and social media, working on several local and EU projects. He organized several workshops, special interest groups, and tutorials at international conferences, and teaches a course on HumanComputer Interaction for master students of the faculties of Social Sciences and Economy, and students of the GroepT Engineering school. David Geerts is member of the IFIP TC14 WG6 on Entertainment Computing, is co-founder and chair of the Belgian ACM SIGCHI.be chapter, is part of the EuroITV steering committee and was program chair of the EuroITV2009 conference on interactive television. 